\title{
ABSOLUTE RIESZ SUMMABILITY OF FOURIER SERIES. I
}

\author{
G. D. DIKSHIT AND C. S. REES
}

\begin{abstract}
In this paper we prove some theorems on the absolute summability of Fourier series which connect diverse $|C, \gamma|$ results such as Bosanquet's classical theorem (1936), Mohanty (1952), and Ray (1970) and the recent $\left|R, \exp \left((\log \omega)^{\beta+1}\right), \gamma\right|$ result of Nayak (1971).

It is also shown that in some sense some of the conclusions of the paper are the best possible.
\end{abstract}

1.1. A series $\Sigma U_{n}$ is said to be absolutely summable by the Riesz method of 'type' $\exp \left((\log \omega)^{\beta+1}\right), \beta>0$, and 'order' $r, r>0$, and written

$$
\sum U_{n} \in\left|R, \exp \left((\log \omega)^{\beta+1}\right), r\right|,
$$

if, with $e(\omega) \equiv \exp \left((\log \omega)^{\beta+1}\right)$,

$$
\int_{A}^{\infty} \frac{e^{\prime}(\omega)}{(e(\omega))^{r+1}}\left|\sum_{n<\omega}\{e(\omega)-e(n)\}^{r-1} e(n) U_{n}\right| d \omega<\infty,
$$

where $A$ is some positive constant. In the case $\beta=0$, the method $|R, e(\omega), r| \equiv$ $|R, \omega, r|$ is known to be equivalent to the Cesàro method $|C, r|$.

1.2. Suppose $f \in L(-\pi, \pi)$ where $f$ is a $2 \pi$-periodic function and let

$$
f(x) \sim \frac{1}{2} a_{0}+\sum_{1}^{\infty}\left(a_{n} \cos n x+b_{n} \sin n x\right) \equiv \sum A_{n}(x) .
$$

We assume, as we may, that $a_{0}=0=A_{0}$.

We use the following notations:

$\phi(t)=\frac{1}{2}\{f(x+t)+f(x-t)\}-s$, for suitably chosen $s=s(x)$,

$e(\omega)=\exp \left\{(\log \omega)^{\beta+1}\right\}, \beta>0$,

$F(\omega, t)=\Sigma_{n<\omega}\{e(\omega)-e(n)\}^{\gamma-1} e(n) n^{-1}(\log n)^{\delta} \sin n t$,

$Q(\omega)=\{e(\omega)-e(m)\}^{\gamma-1} e(m) m^{-1}(\log m)^{\delta}$, where $m$ is an integer such that $m<$ $\omega<m+1$.

Unless otherwise specified, in what follows we use " $\Sigma$ " to denote " $\Sigma_{n=2}^{\infty}$ " and also $\Sigma_{n<\omega}$ to denote $\Sigma_{n=2}^{[m]} . k$ is a suitable constant, while $K, K_{1}, K_{2}, \ldots$ denote absolute constants, possibly different at each occurrence.

Received by the editors February 26, 1980; presented to the Society, January 7, 1981.

1980 Mathematics Subject Classification. Primary 42A28, 40F05; Secondary 42A24, 40G99.

Key words and phrases. Fourier series, absolute Riesz summability, absolute Cesàro summability, absolute convergence, function of bounded variation, totally regular methods. 


\subsection{Theorems.}

TheOREM I. Let $\alpha, \beta, \delta$ and $\eta$ be real numbers such that $\alpha>0$ and either (i) $\beta=0=\eta, \delta<0$, or (ii) $\delta<\eta$ and $\beta$ is any positive number however large. Then

$$
\phi(t)\left(\log \frac{k}{t}\right)^{\eta} \in B V(0, \pi) \Rightarrow \sum A_{n}(x)(\log n)^{\delta} \in\left|R, \exp (\log \omega)^{\beta+1}, \alpha\right| .
$$

The result is the best possible in the sense that ' $\delta<\eta$ ' may not be replaced by ' $\delta<\eta$ ' if $\beta \neq 0, \eta \neq 0$. For, take $k=2 \pi$ and consider $\phi(t)=(\log (2 \pi / t))^{-\eta}$. Then $A_{n}(x) \sim(-\eta) n^{-1}(\log n)^{-\eta-1} \quad\left(\right.$ see Lemma 1 , below), and $\sum A_{n}(x)(\log n)^{\eta} \approx$ $-\eta \Sigma(n(\log n))^{-1}$ cannot be summable by a totally regular method. However in our next theorem, we show that if we strengthen somewhat the requirement on the function $\phi$ we can (in the case $\beta=0$ ) allow $\delta=\eta$.

THEOREM II. Let $\gamma>0, \beta>0$ and $\delta$ and $\eta$ be real numbers such that (i) $\delta<\eta-\beta \gamma /(\gamma+1)$, when $0<\gamma<1$, or (ii) $\delta<\eta-\beta / 2$ when $\gamma>1$. Suppose that $\int_{0}^{\pi}(\log (k / t))^{\eta}|d \phi(t)|<\infty$. Then

$$
\sum A_{n}(x)(\log n)^{\delta} \in\left|R, \exp (\log \omega)^{\beta+1}, \gamma\right| .
$$

2.2. Remarks. (i) The case $\beta=0$ and $\eta=0$ of either of the two theorems contains the basic result of Bosanquet [1] for summability $|C, \alpha|, \alpha>0$.

(ii) The case $\eta=-1$ of Theorem I improves upon Theorem $A$ below, due to Nayak. Nayak discussed only the case $\alpha=1$ and even there he had to take $\delta=-((\beta / 2)+1)$ and not just ' $\delta<-1$ ':

Theorem A [6]. Let $\beta>0$. If $\phi(t) / \log (k / t) \in B V(0, \pi)$, then

$$
\sum A_{n}(x)(\log n)^{-((\beta / 2)+1)} \in|R, e(\omega), 1| \text {. }
$$

(iii) Theorem II extends a theorem due to Ray [7, Theorem 1] on Cesàro summability of Fourier series. Ray's result corresponds to the case $\beta=0, \eta=\delta<$ 0 .

(iv) The case $\beta=0, \eta=1$ of Theorem II contains a result of Mohanty [5].

(v) We note that Theorem II furnishes a better result than Theorem I only when $\beta=0$ (cf. $\$ 3.2$, the proof of Theorem I).

(vi) We are thankful to the referee for pointing out that it follows from inequality (10) below that the hypothesis $\phi(t)(\log (k / t))^{\eta} \in B V(0, \pi)$ implies the stronger result $\sum A_{n}(x)(\log n)^{\delta}$ is absolutely convergent for $\delta<-1$ and $\delta<\eta-1$. Note however that $\delta<-1$ may not be replaced by $\delta<-1$. For, consider the even, $2 \pi$-periodic function $\phi$ defined by $\phi(t)=(\pi / 2) x_{[\pi / 2, \pi]}$ for $t \in[0, \pi]$. Then

$$
\phi(t) \sim \frac{\pi}{4}+\sum_{1}^{\infty} \frac{(-1)^{n} \cos (2 n-1) t}{2 n-1} \equiv \sum_{0}^{\infty} A_{\nu}(t) .
$$

For this series we find that $\Sigma A_{n}(0)(\log n)^{-\delta}$ is not absolutely convergent if $\delta=-1$, while $(\log (k / t))^{\eta} \phi(t) \in B V(0, \pi)$ for any $\eta$. 
2.3. For the proof of Theorem I we first prove the following theorem:

TheORem III. Let $\beta, \gamma, \delta$ and $\eta$ be real numbers with $\gamma>0$. Suppose $\beta>0$ unless $\eta=0$ and then take $\beta>0$; and let either (i) $\delta<\eta-\beta \gamma /(\gamma+1)$ when $0<\gamma<1$, or (ii) $\delta<\eta-\beta / 2$, when $\gamma>1$. Then

$$
\phi(t)(\log (k / t))^{\eta} \in B V(0, \pi) \Rightarrow \sum A_{n}(x)(\log n)^{\delta} \in\left|R, \exp (\log \omega)^{\beta+1}, \gamma\right| .
$$

2.4. We need the following lemmas:

LEMMA 1 (SEE [7]). $\int_{0}^{\pi}(\log (2 \pi / t))^{b} \cos n t d t \sim(\pi / 2) b n^{-1}(\log n)^{b-1}$, for all real $b$.

LEMMA 2. Let $\gamma>0, \beta>0$ and also $\delta>0$ if $\beta=0$. Let $m$ be an integer such that $m<\omega<m+1$. For $\omega \rightarrow \infty$ and $0<t<\pi$, we have

$$
|F(\omega, t)|<\left\{\begin{array}{l}
K t \omega(\log \omega)^{\delta-\beta} e^{\gamma}(\omega)+\operatorname{tm} Q(\omega), \text { for } 0<\gamma<1, \\
K t \omega(\log \omega)^{\delta-\beta} e^{\gamma}(\omega), \text { for } \gamma>1 ;
\end{array}\right.
$$

and for $\omega>(k / t)+2$ and $0<\gamma<1$

$$
|F(\omega, t)|<K t^{-\gamma} \omega^{-\gamma} e^{\gamma}(\omega)(\log \omega)^{\beta \gamma}\left\{(\log \omega)^{\delta-\beta}+(\log (\omega-k / t))^{\delta-\beta}\right\}+Q(\omega)
$$

and for $\omega>(k / t)+2$ and $\gamma>1$

$$
|F(\omega, t)|<K t^{-1} \omega^{-1} e^{\gamma}(\omega)(\log \omega)^{\delta}
$$

Proof of Lemma 2. Let $V(x) \equiv V(\omega, x)=\{e(\omega)-e(x)\}^{\gamma-1}$. Then

$$
|F(\omega, t)|<t \sum_{n<\omega} V(n) e(n)(\log n)^{\delta}
$$

Take $N$ an integer such that $e(u)(\log u)^{\delta}$ is increasing for $u>N$. For $\gamma>1$ and $m>N$

$$
\begin{aligned}
& \sum_{n=N}^{m} V(n) e(n)(\log n)^{\delta} \\
&=-\int_{N}^{m}\left(\sum_{n<x} e(n)(\log n)^{\delta}\right) V^{\prime}(x) d x+V(m) \sum_{n=N}^{m} e(n)(\log n)^{\delta} \\
&<-\int_{N}^{m} V^{\prime}(x) \int_{N}^{x} e(u)(\log u)^{\delta} d u d x-\int_{N}^{m} e(x)(\log x)^{\delta} V^{\prime}(x) d x \\
&+V(m) \int_{N}^{m} e(x)(\log x)^{\delta} d x+V(m) e(m)(\log m)^{\delta} \\
&= \int_{N}^{m} V(x) e(x)(\log x)^{\delta} d x+V(N) e(N)(\log N)^{\delta} \\
&+\int_{N}^{m} V(x) e(x)(\log x)^{\delta}\left(\frac{1}{x}\left\{(\beta+1)(\log x)^{\beta}+\delta(\log x)^{-1}\right\}\right) d x, \quad \text { on integrating by parts, } \\
&< K_{1} \int_{N}^{\omega}\{e(\omega)-e(x)\}^{\gamma-1} \frac{e(x)(\beta+1)(\log x)^{\beta}}{x} \cdot x(\log x)^{\delta-\beta} d x \\
&+K_{2} e^{\gamma-1}(\omega) \\
&< K \omega(\log \omega)^{\delta-\beta} e^{\gamma}(\omega) .
\end{aligned}
$$


For $0<\gamma<1$, and $m>N$ we get

$$
\begin{aligned}
\sum_{n=N}^{m} V(n) e(n) & (\log n)^{\delta} \\
& <\int_{N}^{\omega}\{e(\omega)-e(x)\}^{\gamma-1} e(x)(\log x)^{\delta} d x+V(m) e(m)(\log m)^{\delta} \\
& <K \omega(\log \omega)^{\delta-\beta} e^{\gamma}(\omega)+m Q(\omega) .
\end{aligned}
$$

Also $\Sigma_{n<N} V(n) e(n)(\log n)^{\delta}<K e^{\gamma-1}(\omega)$. This proves (1). Note that when $\gamma>1$, the second term in (1) is dominated by the first.

To prove (2) we first take up the case $0<\gamma<1$. Let $\omega_{1}=[\omega-k / t]$ and $N$ be an integer such that the functions (i) $e(x) x^{-1}(\log x)^{\delta}$, (ii) $e^{\prime}(x)$ and (iii) $\left(e^{\prime}(x)\right)^{\gamma}(\log x)^{\delta-\beta}$ are nondecreasing for $x>N$. Let

$$
F(\omega, t)=\sum_{n=2}^{N}+\sum_{N+1}^{\omega_{1}}+\sum_{\omega_{1}+1}^{m}=S_{1}+S_{2}+S_{3} \text {, say. }
$$

In the case $\omega_{1}<N, S_{2}$ is zero and the lower limit in $S_{3}$ is $(N+1)$.

Now

$$
\begin{aligned}
\left|S_{1}\right| & <K\{e(\omega)-e(N)\}^{\gamma-1}<K e^{\gamma-1}(\omega), \\
\left|S_{2}\right| & <K\left\{e(\omega)-e\left(\omega_{1}\right)\right\}^{\gamma-1} e\left(\omega_{1}\right) \omega_{1}^{-1}\left(\log \omega_{1}\right)^{\delta} \max _{N<a<b<\omega}\left|\sum_{a}^{b} \sin n t\right| \\
& <K t^{-1}\left\{\left(\omega-\omega_{1}\right) e^{\prime}\left(\omega^{*}\right)\right\}^{\gamma-1} e\left(\omega_{1}\right) \omega_{1}^{-1}\left(\log \omega_{1}\right)^{\delta} \\
& \quad \text { for some } \omega^{*}, \omega^{*} \in\left(\omega_{1}, \omega\right), \\
& <K t^{-1} t^{-(\gamma-1)}\left\{e^{\prime}\left(\omega_{1}\right)\right\}^{\gamma-1} e\left(\omega_{1}\right) \omega_{1}^{-1}\left(\log \omega_{1}\right)^{\delta} \\
& <K t^{-\gamma} \omega^{-\gamma} e^{\gamma}(\omega)(\log \omega)^{\beta(\gamma-1)+\delta .}
\end{aligned}
$$

If $\delta>\beta$,

$$
\begin{aligned}
\left|S_{3}\right|< & \int_{\omega_{1}}^{\omega}\{e(\omega)-e(u)\}^{\gamma-1} e(u) u^{-1}(\log u)^{\delta} d u \\
& +\{e(\omega)-e(m)\}^{\gamma-1} e(m) m^{-1}(\log m)^{\delta} \\
< & K(\log \omega)^{\delta-\beta}\left\{e(\omega)-e\left(\omega_{1}\right)\right\}^{\gamma}+Q(\omega) \\
& <K t^{-\gamma} \omega^{-\gamma}(\log \omega)^{\delta+\beta(\gamma-1)} e^{\gamma}(\omega)+Q(\omega)
\end{aligned}
$$

and if $\delta<\beta$,

$$
\begin{aligned}
\left|S_{3}\right|< & K(\log (\omega-k / t))^{\delta-\beta}\left\{e(\omega)-e\left(\omega_{1}\right)\right\}^{\gamma} \\
& +\{e(\omega)-e(m)\}^{\gamma-1} e(m) m^{-1}(\log m)^{\delta} \\
\leqslant & K t^{-\gamma} \omega^{-\gamma}(\log \omega)^{\beta \gamma}(\log (\omega-k / t))^{\delta-\beta} e^{\gamma}(\omega)+Q(\omega) .
\end{aligned}
$$

When $\gamma>1$ obviously

$$
|F(\omega, t)|<K t^{-1}\{e(\omega)-e(2)\}^{\gamma-1} e(\omega) \omega^{-1}(\log \omega)^{\delta} .
$$

Collecting the results from (3) to (6) completes the proof of the lemma. 
LemMA 3. Suppose that $\gamma>0$ and $c>0$. If $\Sigma U_{n} \in|C, \gamma|$ then

$$
\sum U_{n}(\log n)^{-c} \in|C, \gamma|
$$

This is a particular case of the much more general Anderson-Bosanquet-ChowPeyerimhoff Theorem [2] on summability factors of absolute Cesàro summability.

LEMMA 4 [7]. Let $\alpha>0$ and $\eta<0$. If (i) $\phi(t)(\log (k / t))^{\eta} \in B V(0, \pi)$, and (ii) $\int_{0}^{\pi}|\phi(t)| / t(\log (k / t))^{1-\eta} d t<\infty$ then $\sum A_{n}(x)(\log n)^{\eta}$ is summable $|C, \alpha|$.

LEMMA 5. Let $\eta<0$. Then (i) and (ii) hold if and only if (iii) holds, where

(i) $\phi(t)(\log (k / t))^{\eta} \in B V(0, \pi)$,

(ii) $(\log (k / t))^{\eta-1} \phi(t) / t \in L(0, \pi)$, and

(iii) $\int_{0}^{\pi}(\log (k / t))^{\eta}|d \phi(t)|<\infty$.

Proof. Since

$$
d\left\{(\log (k / t))^{\eta} \phi(t)\right\}=(\log (k / t))^{\eta} d \phi(t)-\eta(\log (k / t))^{\eta-1} \phi(t) d t / t
$$

then (iii) follows from (i) and (ii).

Conversely, we have, for $0<t<\pi,|\phi(t)|<|\phi(\pi)|+\int_{t}^{\pi}|d \phi(u)|$. Hence

$$
\begin{aligned}
\int_{0}^{\pi}(\log (k / t))^{\eta-1} \frac{|\phi(t)|}{t} d t \\
\quad<|\phi(\pi)| \int_{0}^{\pi}(\log (k / t))^{\eta-1} \frac{d t}{t}+\int_{0}^{\pi} \int_{t}^{\pi}|d \phi(u)|(\log (k / t))^{\eta-1} \frac{d t}{t} \\
<K+\int_{0}^{\pi} \int_{0}^{u}(\log (k / t))^{\eta-1} \frac{d t}{t}|d \phi(u)| \\
\quad=K+\frac{1}{|\eta|} \int_{0}^{\pi}(\log (k / u))^{\eta}|d \phi(u)|
\end{aligned}
$$

Thus (ii) follows (iii), and then (i) follows from (ii) and (iii) with the aid of (*).

3.1. Proof of Theorems. We shall combine the proofs for Theorems II and III.

For Theorem II in the case $\beta=0, \delta=\eta<0$, the result follows from Lemmas 4 and 5 , and then for $\beta=0, \delta<\eta<0$, the result follows by Lemma 3. Simiarly for $\beta=0, \delta<0<\eta$, the result follows from the case $\beta=0,0<\delta<\eta$, by Lemma 3 . Hence it remains to consider the cases (i) $\beta=0, \eta>\delta>0$ and (ii) $\beta>0$. Similarly, for Theorem III the case $\beta=0=\eta, \delta<0$, follows from the case $\beta=0$, $\eta=0=\delta$, after Lemma 3 . Hence we need to consider this theorem also only for the cases (iii) $\beta=0=\eta=\delta$ and (iv) $\beta>0$. 
To begin with for Theorem III, we note that, for $\boldsymbol{n}>1$,

$$
\begin{aligned}
\frac{\pi}{2} A_{n}(x)= & \int_{0}^{\pi} \phi(t) \cos n t d t \\
= & {\left[\phi(t)(\log (k / t))^{\eta} \int_{0}^{t}(\log (k / u))^{-\eta} \cos n u d u\right]_{0}^{\pi} } \\
& -\int_{0}^{\pi}\left\{\int_{0}^{t}(\log (k / u))^{-\eta} \cos n u d u\right\} d\left\{\phi(t)(\log (k / t))^{\eta}\right\} \\
= & \phi(\pi)(\log (k / \pi))^{\eta} \int_{0}^{\pi}(\log (k / u))^{-\eta} \cos n u d u \\
& -\frac{1}{n} \int_{0}^{\pi}\left\{\sin n t(\log (k / t))^{-\eta}-\eta \int_{0}^{t} \frac{\sin n u}{u}(\log (k / u))^{-\eta-1} d u\right\} \\
& \cdot d\left\{\phi(t)(\log (k / t))^{\eta}\right\} .
\end{aligned}
$$

For $t>1 / n$ and $\eta \neq 0$

$$
\begin{aligned}
\left|\int_{0}^{t} \sin n u(\log (k / u))^{-\eta-1} u^{-1} d u\right|=\left|\int_{0}^{1 / n}+\int_{1 / n}^{t}\right| \\
<n \int_{0}^{1 / n}(\log (k / u))^{-\eta-1} d u+\left|\left[-\cos n u(\log (k / u))^{-\eta-1} n^{-1} u^{-1}\right]_{1 / n}^{t}\right| \\
\quad+\frac{1}{n}\left|\int_{1 / n}^{t} \cos n u \frac{d}{d u}\left\{u^{-1}(\log (k / u))^{-\eta-1}\right\}\right| \\
<K(\log n)^{-\eta-1}
\end{aligned}
$$

and for $0<t<1 / n$ and $\eta \neq 0$

$$
\begin{aligned}
\left|\int_{0}^{t} u^{-1}(\log (k / u))^{-\eta-1} \sin n u d u\right| & <n \int_{0}^{1 / n}(\log (k / u))^{-\eta-1} d u \\
& <K(\log n)^{-\eta-1} .
\end{aligned}
$$

Thus, since $\int_{0}^{\pi}\left|d\left\{\phi(t)(\log k / t)^{\eta}\right\}\right|$ is finite, from (7)-(9) and Lemma 1, we get

$$
\left|\frac{\pi}{2} A_{n}(x)+\frac{1}{n} \int_{0}^{\pi} \sin n t(\log (k / t))^{-\eta} d\left\{\phi(t)(\log (k / t))^{\eta}\right\}\right|<K n^{-1}(\log n)^{-\eta-1} \text {. }
$$

For $\eta=0$, we see by (7), the right-hand side in (10) is zero.

Since $\Sigma n^{-1}(\log n)^{\delta-\eta-1}, \eta-\delta>0$, is absolutely convergent, to prove that $\sum A_{n}(x)(\log n)^{\delta} \in|R, e(\omega), \gamma|$ (whether $\eta=0$ or not), it is enough to show that the integral

$$
\begin{aligned}
\int_{2}^{\infty}(\log \omega)^{\beta} \omega^{-1} e^{-\gamma}(\omega) \mid & \sum_{n<\omega}\{e(\omega)-e(n)\}^{\gamma-1} e(n)(\log n)^{\delta} n^{-1} \\
& \int_{0}^{\pi} \sin n t(\log (k / t))^{-\eta} d\left\{\phi(t)(\log (k / t))^{\eta}\right\} \mid d \omega
\end{aligned}
$$

is convergent. As $\phi(t)(\log (k / t))^{\eta} \in B V(0, \pi)$, it is sufficient to show that

$$
\int_{2}^{\infty}(\log \omega)^{\beta} \omega^{-1} e^{-\gamma}(\omega)|F(\omega, t)| d \omega=O\left((\log (k / t))^{\eta}\right)
$$


uniformly in $t, 0<t<\pi$. Let

$$
I(t)=\int_{2}^{\infty}(\log \omega)^{\beta} \omega^{-1} e^{-\gamma}(\omega)|F(\omega, t)| d \omega .
$$

For the proof for Theorem II we note that, for $\boldsymbol{n}>1$,

$$
\frac{\pi}{2} A_{n}(x)=\int_{0}^{\pi} \phi(t) \cos n t d t=-(1 / n) \int_{0}^{\pi} \sin n t d \phi(t)
$$

As $\int_{0}^{\pi}(\log (k / t))^{\eta}|d \phi(t)|$ is finite, in this case also it is sufficient to show that the requirement (11) is fulfilled, that is

$$
I(t)=O\left((\log (k / t))^{\eta}\right), \text { uniformly in } t, 0<t<\pi .
$$

Let $\tau=(k / t)(\log (k / t))^{\beta \gamma^{\prime}}+2$, where $\gamma^{\prime}=\min \left(\gamma /(\gamma+1), \frac{1}{2}\right)$, and

$$
I(t)=\int_{2}^{\tau}+\int_{\tau}^{\infty}=I_{1}(t)+I_{2}(t), \text { say. }
$$

After the estimates in (1) we get

$$
\begin{aligned}
I_{1}(t) & <K_{1} t \int_{2}^{\tau}(\log \omega)^{\delta} d \omega+K_{2} t \int_{2}^{\tau}(\log \omega)^{\beta} \omega^{-1} e^{-\gamma}(\omega) m Q(\omega) d \omega \\
& <K_{1} t \tau(\log \tau)^{\delta}+K_{2} t \tau^{1-\gamma}(\log \tau)^{\delta+\beta \gamma} \\
& =O\left((\log (k / t))^{\eta}\right), \text { uniformly in } t, 0<t<\pi,
\end{aligned}
$$

since, for $0<\gamma<1$,

$$
\begin{aligned}
\int_{2}^{\tau}(\log \omega)^{\beta} \omega^{-1} e^{-\gamma}(\omega) m Q(\omega) d \omega \\
\\
<\sum_{m<\tau} \int_{m}^{m+1}\{e(\omega)-e(m)\}^{\gamma-1} \frac{(\log \omega)^{\beta}}{\omega e^{\gamma}(\omega)} e(m) \cdot(\log m)^{\delta} d \omega \\
\quad<K \sum_{m<\tau}(\log m)^{\delta} e^{-\gamma}(m+1)\{e(m+1)-e(m)\}^{\gamma} \\
<K \sum_{m<\tau} m^{-\gamma}(\log m)^{\delta+\beta \gamma} .
\end{aligned}
$$

For $I_{2}(t)$, we first consider the case $0<\gamma<1$. Using the order estimates from (2)(i) and proceeding as for (15) and (16),

$$
\begin{aligned}
I_{2}(t)< & K_{1} t^{-\gamma} \int_{\tau}^{\infty}(\log \omega)^{\beta(\gamma+1)} \omega^{-\gamma-1}\left\{(\log \omega)^{\delta-\beta}+(\log (\omega-k / t))^{\delta-\beta}\right\} d \omega \\
& +K_{2} \int_{\tau}^{\infty}(\log \omega)^{\beta} \omega^{-1} e^{-\gamma}(\omega) Q(\omega) d \omega \\
< & K_{1} t^{-\gamma} \tau^{-\gamma}(\log \tau)^{\beta(\gamma+1)}\left\{(\log \tau)^{\delta-\beta}+(\log (\tau-k / t))^{\delta-\beta}\right\} \\
& +K_{2} \sum_{[\tau]}^{\infty} m^{-\gamma-1}(\log m)^{\delta+\beta \gamma} \\
= & O\left((\log (k / t))^{\eta}\right), \text { uniformly in } t, 0<t<\pi .
\end{aligned}
$$


For $\gamma>1$, the order estimates in (2)(ii) give

$$
\begin{aligned}
I_{2}(t) & <K t^{-1} \int_{\tau}^{\infty}(\log \omega)^{\delta+\beta} \omega^{-2} d \omega \\
& =O\left((\log (k / t))^{\eta}\right), \text { uniformly in } t, 0<t<\pi .
\end{aligned}
$$

This completes the proof for Theorems II and III.

3.2. Proof of Theorem I. Note that $\delta<\eta$ implies that $\delta<\eta-\beta \gamma /(\gamma+1)$, for all arbitrarily small positive $\gamma, 0<\gamma<1$. We choose $\gamma$ such that it is also less than $\alpha$. Now by Theorem III, $\sum A_{n}(x)(\log n)^{\delta} \in|R, e(\omega), \gamma|$. Hence, by the First Theorem of Consistency for absolute Riesz summability (see [3, \$1.9])

$$
\sum A_{n}(x)(\log n)^{\delta} \in|R, e(\omega), \alpha| \text {. }
$$

4. We obtain the following corollary to our Theorems I and II.

COROLlARY. Let $\alpha>0$ and $\delta$ and $\eta$ be real numbers. If either

(I) $\eta \neq 0, \delta<\eta$ and $\phi(t)(\log (k / t))^{\eta} \in B V(0, \pi)$, or

(II) $0<\eta, \delta<\eta$ and $\int_{0}^{\pi}(\log (k / t))^{\eta}|d \phi(t)|$ is finite

then $\sum A_{n}(x)(\log n)^{\delta} \in|C, \alpha|$.

Part (I) of the corollary follows from Theorem I after a 'Second Theorem of Consistency' for absolute Riesz summability (see [4, Theorem 2]).

We are thankful to the referee for his detailed helpful comments, which have improved the paper significantly.

\section{REFERENCES}

1. L. S. Bosanquet, Note on the absolute summability $(C)$ of a Fourier series, J. London Math. Soc. 11 (1936), 11-15.

2. L. S. Bosanquet and H. C. Chow, Some remarks on convergence and summability. factors, J. London Math. Soc. 32 (1957), 73-82.

3. K. Chandrasekharan and S. Minakshisundaram, Typical means, Oxford Univ. Press, New York, 1952.

4. B. Kuttner, On the 'Second Theorem of Consistency' for absolute Riesz summability, Proc. London Math. Soc. (3) 29 (1974), 17-32.

5. R. Mohanty, Absolute Cesàro summability of a series associated with a Fourier series, Bull. Calcutta Math. Soc. 44 (1952), 152-154.

6. M. K. Nayak, On the absolute summability and convergence of Fourier series and associated series, Proc. Cambridge Philos. Soc. 70 (1971), 421-433.

7. B. K. Ray, On the absolute summability of some series related to a Fourier series, Proc. Cambridge Philos. Soc. 67 (1970), 29-45.

Department of Mathematics, University of Auckiand, Auckiand, New Zealand

Department of Mathematics, University of New Orleans, New Orleans, lousiana 70122 